\title{
Smart Mirror using Artificial Intelligence
}

\author{
Pranit Ghag \\ Student, Electronics and \\ Telecommunication \\ Dwarkadas J. Sanghvi College of \\ Engineering \\ Mumbai, India
}

\author{
Vineet Kanetkar \\ Student, Electronics and \\ Telecommunication \\ Dwarkadas J. Sanghvi College of \\ Engineering \\ Mumbai, India
}

\author{
Revathi A. S. \\ Assistant Professor, Electronics \\ and Telecommunication \\ Dwarkadas J. Sanghvi College of \\ Engineering \\ Mumbai, India
}

\begin{abstract}
The purpose of this paper is to use a common day-to-day object and transform it into an intelligent device using a raspberry pi module. This results in a smart mirror which is an embedded device with artificial intelligence. The framework to be developed will have face recognition for authentication and security purposes, given the increasing demand for protection of user attributes and profile. Further, the mirror will respond to voice commands and display weather updates, news and maps on the screen. This is analogous to Alexa of Amazon, Cortana of Microsoft and Siri of Apple. This device has a wide reach and can be employed in advertising and marketing. It has important applications in the automotive industry as it can be used in place of a rear-view mirror to display important data to the person driving the car.
\end{abstract}

\section{Keywords}

Artificial intelligence, framework, authentication, advertising

\section{INTRODUCTION}

There have been many efforts by developers and engineers by constantly innovating and creating solutions to optimize our resources. This drive has produced technologies that have resulted from day to day objects and paraphernalia. It can start at the most basic level and use daily items to make life easy and simple. There are many options available but a mirror is one of the best inanimate objects to start with. We wake up every day and look at our phones for various updates. But instead, a mirror can be employed to delineate weather updates, current news, GPS traffic and usual interaction.

With recent progress in fields like artificial intelligence and machine learning, there has been a growing need for artificial intelligent assistants in automation and industry. Thus, a mirror can be used as an embedded device with human like intelligence. IoT is used effectively to derive the essential data needed to be displayed on the mirror. Internet technologies have been crucial in developing such projects.

The main objective was to develop a project which could be used in numerous applications and combine recent trends such as artificial assistant and embedded technology. Python code has been used for the smart mirror. The rest of the paper is organized as follows. Section 2 talks about the components used and block diagram. Section 3 mentions face recognition, artificial intelligence and GUI creation. Section 4 describes the simulation results. Section 5 explains the conclusion and future scope.

This paper shows how a smart mirror is developed using various embedded components. The mirror is used to display contents from the web. But to do so, it uses infrared sensors. Whenever a person comes in front of the screen, the sensors detect the person and display various information. This project explores the possibility of being used in home automation. Using voice commands, lights can be turned on and off. Music can be played using speech recognition. Thus, use of PIR sensors to detect the presence of humans is done which enables home automation. These are small, inexpensive and low power devices. [1]

Controlling home appliances is a desirable aspect which one would want to make life easier and faster. This is made possible by using a smart mirror and IoT. [8] In India it has gained popularity as the Government of India has set aside USD 15 billion for the Iot industry. Companies such as Apple, Samsung and Alphabet have all contributed and created related products due to the promising USD 79.4 billion generated by this sector in 2014. The basic components required are a monitor, mirror, raspberry-pi and a relay board. It proposes the use of GrovePi to add more than one modules to the existing Pi board. The relay board is used to separate these modules. A 37 module sensor kit can be used along with the raspberry pi to provide a number of features. [2]

This paper illustrates the use of mirror technology in automobiles. It also explores the work of manufacturers in developing Advanced Driver Assistance Systems (ADAS), which help the driver in making quick decisions in critical situations. This project aims at improving safety standards in cars by enhancing the driver's understanding of his or her surroundings. As much as $94 \%$ of traffic crashes can be assigned to a driver. The mirror used is called as Rear-View Stitched Panorama (RSVP). The three mirrors in cars will be replaced by cameras and the real time display of those cameras will be combined into a panoramic display. [3]

\section{COMPONENTS USED \\ Block Diagram}

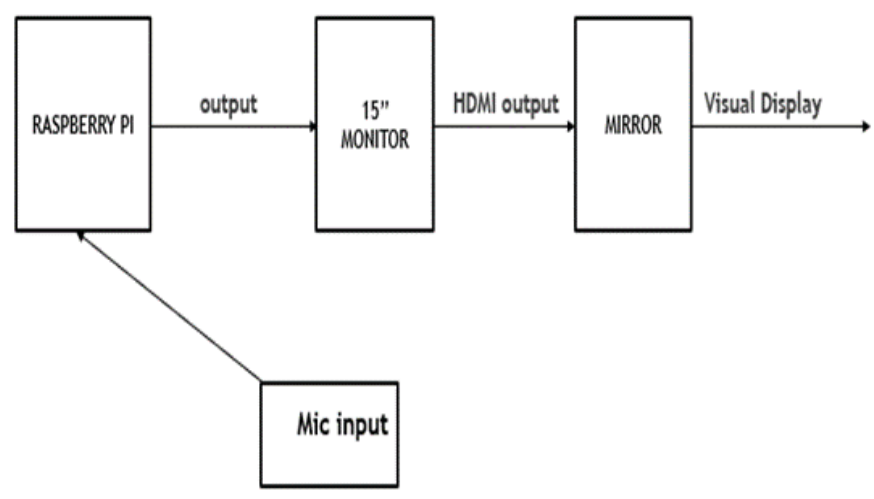

Components 
- $\quad$ Raspberry pi

- Two-way mirror

- Monitor with HDMI connection

- Webcam for face capturing

- Microphone

Raspberry-pi acts as the operating system and runs the Python code. It has a RAM of $1 \mathrm{~GB}$ and supports HDMI connection. The code is transferred to the Monitor from the raspberry pi using an HDMI cable. An LCD monitor is used to display the required data and tools for interactive user communication.

Only authenticated users can have access to the mirror. This is achieved using facial identification. Hence, a webcam is used for tracking user movements and positions. Along with this, a microphone is needed so that instructions or commands could be given to the smart mirror.

A wooden frame must be used to enclose the entire structure. This protects the edges of the mirror, monitor being encapsulated as well as the raspberry pi which runs the code. [4]

\section{PROPOSED WORK}

\subsection{Programming}

Code is written in Python to enable the mirror as an interactive device. Human like intelligence is given by interfacing microphone and a webcam. Libraries like wit.ai were used. Theses libraries obtained tokens and could obtain the information such as weather updates and current news. Python code enabled human like intelligence for the embedded device. The code is loaded into the operating system which is raspberry pi and it controls the entire operation. The code is such that it makes the mirror work as an interactive tool.

\subsection{Face Recognition}

The purpose is to allow access only to the authenticated users. This is achieved using linear binary pattern algorithm. The linear binary pattern cascade classifier helps us to achieve face detection while the local binary pattern histogram is used for identification. [6]

The aim is to capture images using a camera or a webcam and process it using the code written in Python. The LBP focusses on the centre pixel and compares all neighbouring pixels with the centre pixel. Depending on the difference, it sets pixel to a value ' 1 ' if the difference is positive and to a value ' 0 ' if the difference between centre pixel and the neighbouring pixel is negative. The converted 8 bits are arranged into a byte. This is done for all of the pixels covering the face.

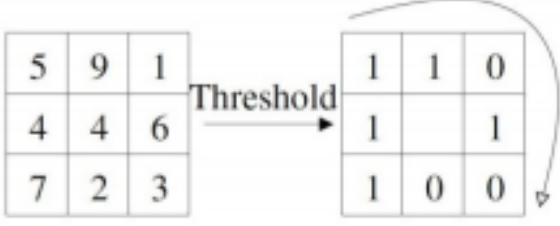

Fig 1. LBP Process

Binary: 11010011

Decimal: 211

Fig 2. Face Recognition Result

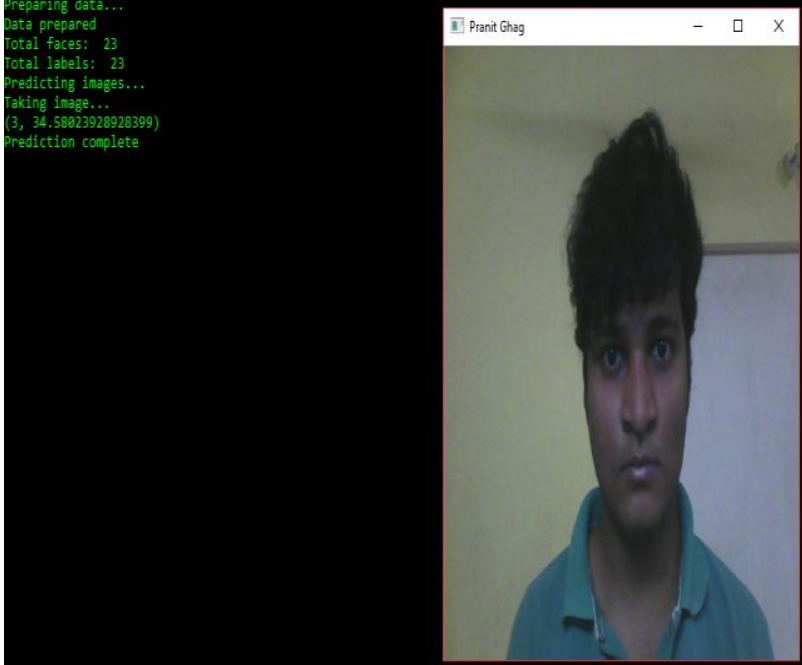

Fig 3. Face Recognition Process

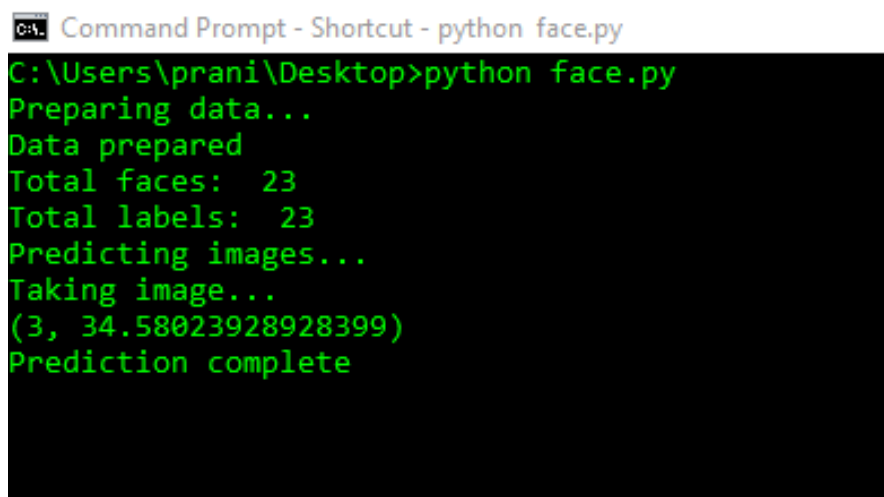

The captured photo is compared with the one in the database and we get the desired result.

\subsection{Artificial Intelligence}

The mirror has been incorporated with smart intelligence to answer human queries. The trigger has been set as "Ok Mirror". After detecting the trigger, the mirror responds to the authenticated user.

For imparting intelligence to the device, we have used intents from wit.ai. Different intents can be created to perform the desired application. The user-end can perform the required task by defining and creating one's own intents. Some common examples are to set an alarm or a reminder, switch on/off the lights in case of home automation and adjust the temperature in a centralized air-conditioned room. Some of the intents used are appreciation, YouTube, greeting, joke and so on.

The mirror is a learning network which can tell you a joke, give you the weather updates, news updates, satellite maps and so on. Instead of looking in one's phone all the time, this mirror does all the job and provides the necessary data. Intents involve the creation of entities. Roles must be defined when there are multiple entities in an intent. For example, for GPS navigation the source and destination should be clearly specified by assigning different roles.

This mirror supports third-party applications by using houndify library created by SoundHound. Houndify provides a number of domains for the user to interact with. Some are Sports, Music Search, Stock Market, Email and so on. Once the user launches a query, the mirror looks into the internet and provides a solution using the various domains. For example, a YouTube video can 
be played on the screen just by voice commands. This deice promotes hands-free applications which is rapidly becoming popular.

\subsection{Graphical User Interface (GUI) creation:}

It is important for any device to convey its message to the user for the specified application. A device can be called 'smart' when it is interactive and is easy to communicate with. This mirror has a GUI which displays user-defined options and the answer to user's voice commands is displayed in the centre. Date and time can be seen in the top left corner with current news at the bottom. Weather updates can be seen in the top right corner. Third-party applications like YouTube and Zomato can be seen on the extreme right-hand side.

\section{SIMULATION RESULTS}

The results were observed for a number of microphone inputs. The system is quick to respond and displays the required information on the screen. This device can be used in the marketing industry due to its strong potential for being a costumer friendly application. The Graphical User Interface provides most of the necessary details that a person looks up for in his smart phone.

Query: "Ok Mirror"

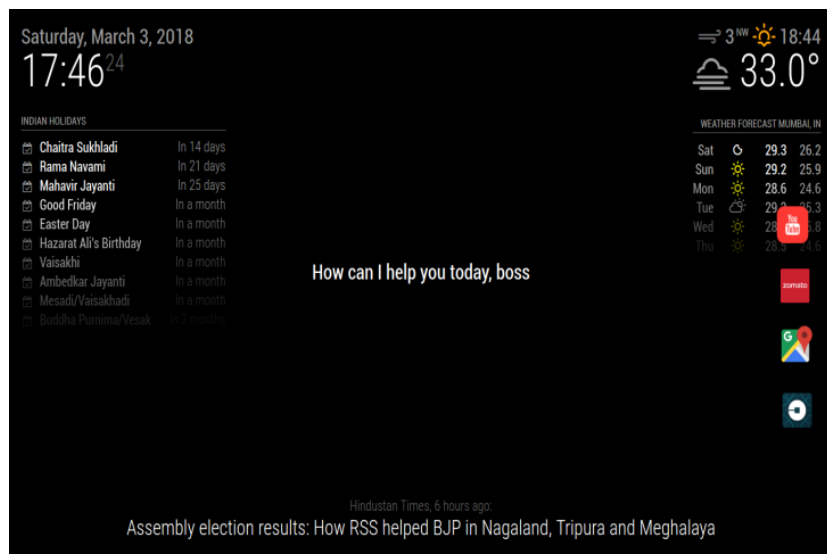

Fig 4. Smart Mirror response after trigger "Ok Mirror" Query: "Show me the satellite map of Mumbai"

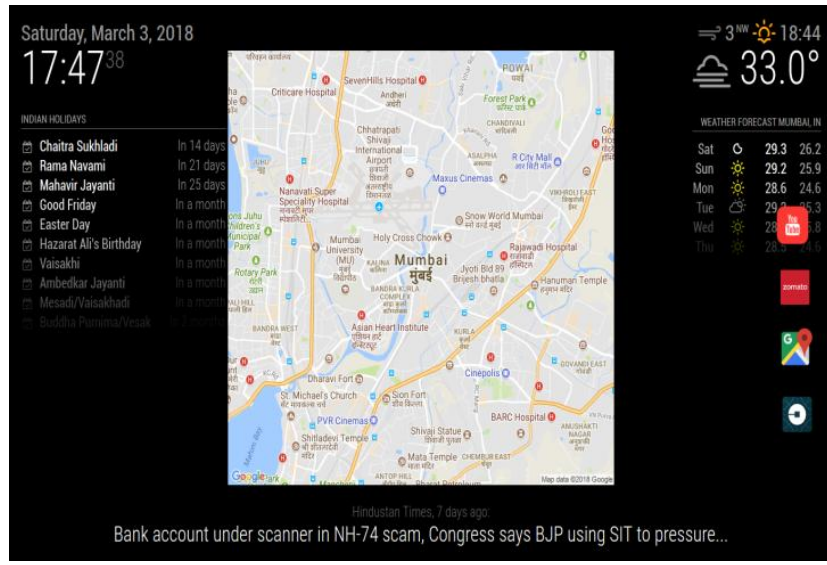

Fig 5. Smart Mirror showing satellite map of Mumbai

\section{Query: Play specific video on YouTube}

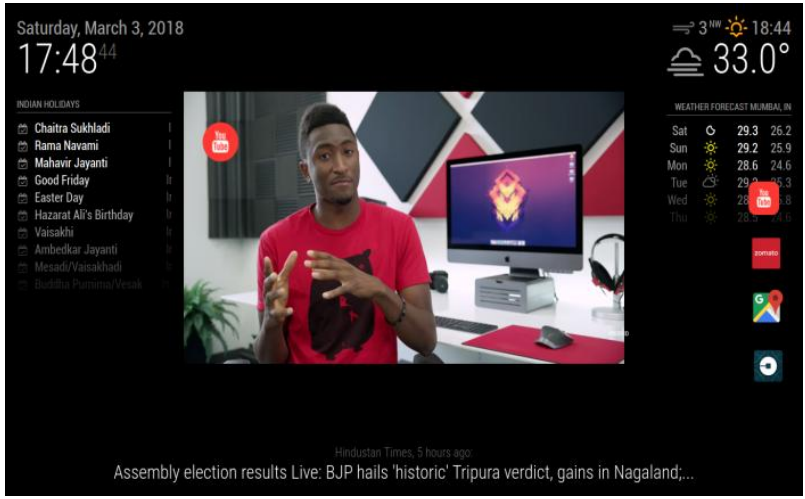

Fig 6. Smart Mirror displaying YouTube

This device can be installed in malls, airports or even business organizations and corporate buildings. With simple voice commands, latest news can be viewed and it can also narrate a joke to lighten the mood in a workplace or any other environment.

The aim is to find a balance between face recognition and artificial intelligence. The accuracy and speed of the user recognition will determine the performance of the mirror as a whole. The speed will determine the hardware requirements and whether raspberry pi is suitable enough to be the operating system of the mirror.

A database is collected and maintained for different users and it can be tested for the mirror. Twelve photos are required for each user in order to have the recognition run properly. The recognised image is displayed along with the name of the person mentioned in the database. Once the recognition, is done, the user can start with his or her voice commands and have the desired result on the screen.

The mirror explores the possibility of including third party applications in the framework. The inclusion of YouTube can be seen in the figures provided. Thus, the mirror is providing us with social contributions when it plays third-party application functions. For videos, a timeout is given after which the video stops playing. This helps us to control the content on the screen and provide a demo of the entire YouTube application. For displaying the news, Really Simple Syndication (RSS) feed is used to deliver the necessary data. However, for third-party applications, external data is made available by using Application Programming Interfaces (API). [7]

\section{CONCLUSION AND FUTURE SCOPE}

Thus, we have developed a mirror with strong intelligence features so as to enable the user as an artificial intelligence assistant. The entire assembly is designed for optimum performance and performs the desired application. With growing demand for intelligent applications, a number of companies have produces their own versions of "smart mirrors".

Several companies have come forward and developed their own versions of Smart Mirrors. The development of intelligent mirrors began around year 2000. Toshiba developed a mirror which was based on gesture control. They aimed at creating personalised mirrors for different applications in the home environment. Microsoft joined the smart mirror industry and introduced a mirror which could be developed as a Do-ItYourself project. Philips and HiMirror developed mirrors which could be used for medical and health related applications. [5]

Thus, over the past two decades the smart mirror industry has developed and grown into a commercial and a strong 
technological influence in the market. Research is still ongoing to integrate health monitoring and make the device completely hands-free. Night-vision cameras can be installed to eliminate the errors in face recognition due to poor lighting conditions and so on. Endless applications can be developed and can be made available to the user-end platform.

\section{REFERENCES}

[1] S. A, Francis. F, Raphel. R, S. S, Porinchu. S, Francis. S, "SMART MIRROR: A Novel Framework for Interactive Display", ICCPCT.

[2] Jose. J, Chakravarthy. R, Jacob. J, Ali. M and D'Souza. S, "Home Automated Smart Mirror as an Internet of Things (IoT) Implementation - Survey Paper”, Vol. 6, Issue 2, February 2017, IJARCCE.

[3] Pan. J, Appia. V, Villareal. J, Weaver. L and Kwon. D, "Rear-Stitched View Panorama: A low-power embedded implementation for smart rear-view mirrors on vehicles", 2017 IEEE Conference on Computer Vision and Pattern Recognition Workshops.
[4] Khanna. V, Vardhan. Y, Nair. D, Pannu. P, "Wesign and development of a smart mirror using raspberry pi", Volume5, Issue-1, Jan.-2017, International Journal Of Electrical, Electronics And Data Communication, ISSN: 2320-2084.

[5] Moskvil. J, “The Intelligent Mirror", June 2017, Norwegian University of Science and Technology.

[6] Akariman. Q, Jati. and Novianty. A, "Face Recognition Based on the Android Device Using LBP Algorithm”, 2015, ICCEREC.

[7] Maheshwari. P, Kaur. M and Anand. S, "Smart Mirror: A Reflective Interface to Maximize Productivity", Volume 166 - No.9, May 2017, International Journal of Computer Applications (0975 - 8887).

[8] Patchava. V, Kandala. H, Babu. P, "A Smart Home Automation Technique with Raspberry Pi using IoT", 2015, International Conference on Smart Sensors and Systems (IC-SSS) 